\title{
TELEVISION SOUND OPERATORS
}

\section{WHO WERE THEY AND WHAT EXACTLY DID THEY DO?}

\author{
Tim Heath \\ Independent Researcher \\ 180 Athelstan Road \\ Southampton, SO19 4DJ \\ United Kingdom \\ timheath91@gmail.com
}

\begin{abstract}
The working practices of below the line television operators is an area of television studies that continues to be under-researched. Despite notable recent efforts, this lack of academic engagement is perhaps at its most pronounced in regards to the sub group of television operators who record, mix, and edit the soundtrack of British television. However, hands on methodologies continue to gain traction in the area of film and television research and, in doing so, create new opportunities to engage with below the line practices and bring into focus the hidden work of production personnel. This article, aims to explore these new methodologies and assess how they can bring new affordances to researchers engaging with communities who's practices are often seen as routine and unremarkable. Focusing specifically on the work of television sound operators this article hopes to add to the growing body of work that sheds light on the practices of sound operators and the skills, codes, and identities that shape their work. By doing so through using hands on methods, it hopes to show the benefits of such approaches to wider television and film research.
\end{abstract}

Keywords: sound, television, hands on history, media archaeology, sound practice, production studies

\section{Introduction}

Outside of the small group of professionals that make up Britain's television sound operators, few give much thought to the processes involved in capturing, editing, and perfecting a programme's soundtrack. The sound of most programming has traditionally functioned to minimise its own presence and to obscure completely the days of intensive skill labour that contributed to its form and content. Whilst the visuals take centre stage the soundtrack passes unnoticed by viewers except in times of extreme error. When such errors occur, the resulting reaction often betrays the widespread lack of understanding about this established and complex profession ${ }^{1}$. Whilst studies of sound 
practice in the American film industry gather pace ${ }^{2}$ the work of their counterparts in the television industry have continued to lag behind.

The aim of this article is twofold. Firstly, it hopes to add to the growing list of academic research into the practices and processes of sound recording, editing, and mixing. Building on the work of scholars focussed on the American film industry, this article aims to extend this analysis into the industrial and technological context and specificities of the British television industry. Secondly, this article will make a methodological argument in favour of a hands-on approach to historical television production studies. In doing so it will argue that to draw out, and give visibility to, televisions hidden production processes we must extend our methodological tools, supplementing established methods with new data which is better equipped to focus on the ordinary and mundane. By doing so, television researchers can gain further understanding not only of production studies, and the processes behind television content, but of televisual form itself.

To meet its first aim, this article will explore the people, practices, and technologies of television sound production throughout the 1960s and 1970s. Focusing on the work of Outside Broadcast (OB) personnel, this article will analyse how the unique environment of OB production shaped the practices of those working on its soundtrack. Furthermore, it offers new insight into some of the numerous, highly complex, technologies used by this group and analysis of how sound operators adapted and shaped their practices around the affordances and limitations of these professional tools. As shall be shown, sound operators of this time were a complex community made up of highly skilled workers with strong hierarchies, bonds, and professional identities.

Throughout this analysis, it shall be demonstrated that hands-on methodologies offer significant new opportunities for historical research into production communities and practices. Where true ethnographic research is not possible, these new methods allow for a richer exploration of the daily, unspectacular, working processes of ordinary, below the line personnel. For such personnel, many of their practices and skills are hidden and largely unspoken, developed through years of on the job training and passed tacitly from one professional to the next. While highly significant in shaping their work and professional identities such practices are hard to capture for those interested in analysing such groups.

Hands on, observational analysis provides greater opportunities to capture these unspoken processes that are so easily missed, overlooked, or distorted by tools such as oral history. As evidenced by Andreas Flickers and Annie Van Den Oever $^{3}$, hands on history allows us not only to discuss but to sense the historical context of an event or technological system. In doing so, television researchers can "become sensitive to everything that evades pure description". When utilised effectively, hands-on methods can act to circumvent some of the pitfalls of traditional methodologies utilised by researchers of production studies and in turn expose the practices and individuals that shape both the industry and its output. Whilst the programmes and products of the British television industry, are well served by scholars in the field of television studies, as evidenced by John Ellis ${ }^{4}$, there is a tendency to analyse the programmes in isolated objects and not as the end result of the practices of dozens, sometimes hundreds, of individuals. Giving visibility to these individuals and the specificities of their practices aids to improve our understanding of televisual form and how processes of production influence television as a medium. Clearly, hands on research offers an opportunity to close the gap between theoretical and practical engagement and avoid the "danger of a reductionist perspective on media technologies, reducing the historical evidence of things to their textual tradition"

2 Katie Quanz, (2015) 'Pro Tools, Playback, and the Value of Postproduction Sound Labor In Canada,' The Velvet Light Trap, 76, 1, 37-48; Helen Hanson, Hollywood Soundscapes; Film Style, Craft \& Production in the Classical Era, BFI Palgrave, 2017.

3 Annie van den Oever and Andreas Fickers, 'Doing Experimental Media Archaeology: Epistemological and Methodological Reflections on Experiments with Historical Objects of Media Technologies,' in Ben Roberts and Mark Goodall, eds, New Media Archaeologies, Amsterdam University Press, 2019, pp. 45-68.

4 John Ellis, 'Theory, Practice and the Importance of Getting Your Hands On,' CST Online, 25 February 2016, https://cstonline.net/theorypractice-and-the-importance-of-getting-your-hands-on-by-john-ellis/

5 Andreas Fickers, 'Experimental Media Archaeology: A Plea for New Directions,' in Annie van den Oever, ed, Technē/Technology Researching Cinema and Media Technologies - Their Development, Use, and Impact, Amsterdam University Press, 2013, pp. 272-27. 
The work of sound operators, and wider production personnel, employed during television's era of vertical integration provide a rich opportunity for new research. Although mostly retired, many of the original operators are able, and willing to actively share their experience. Whilst hard to source and harder still to get working, the original tools of their trade also remain available for researchers who wish to find them. As shall be shown in the following work, hands on methodologies provide an opportunity to make use of these precious resources.

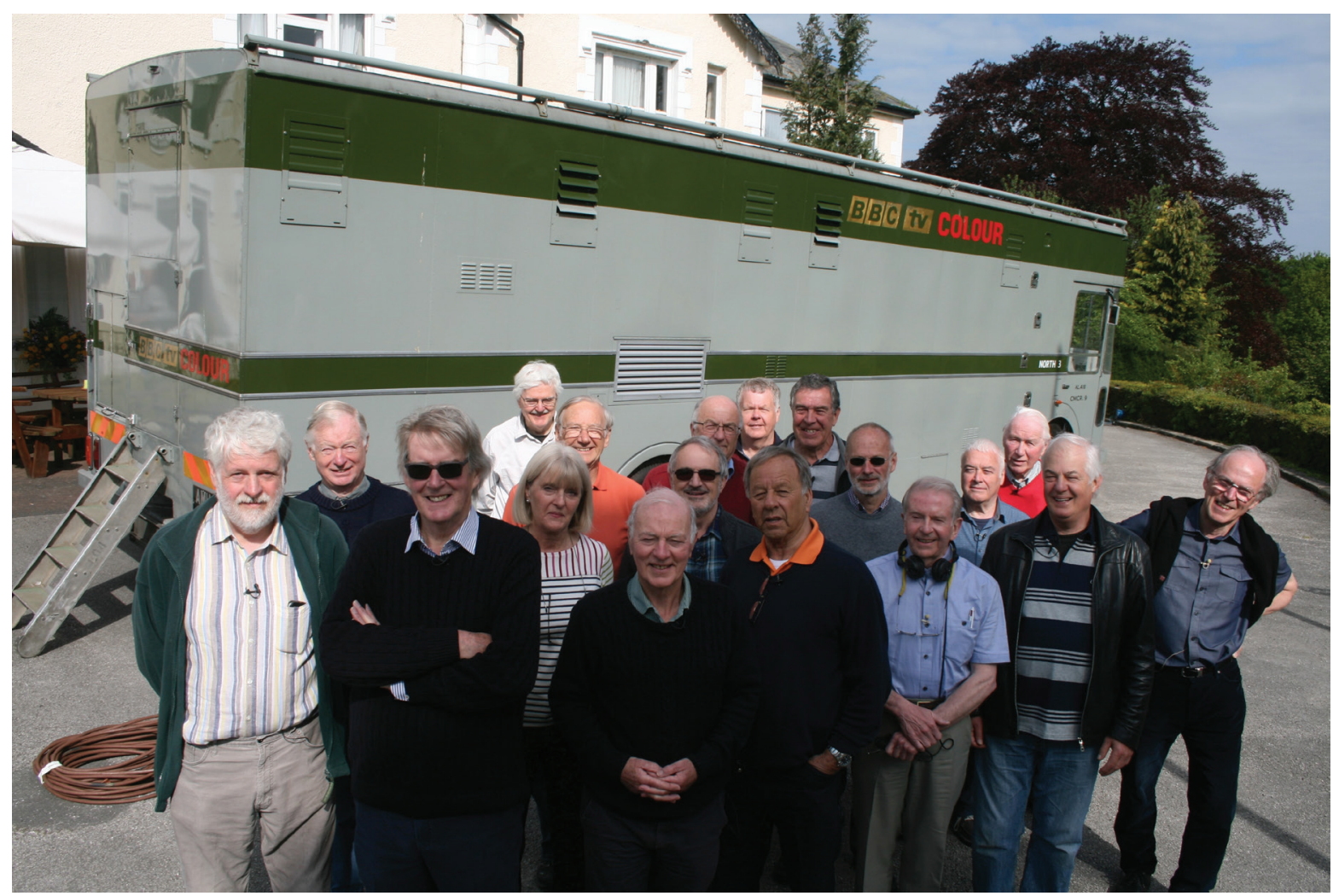

Figure 1. Reunited BBC OB crew.

\section{Hands-On Methodologies in Production Studies}

This research places itself firmly in the realm of production studies. It is interested in the people at the heart of this small sub-community (television sound operators) and, as such, aims it analysis at the micro level of individual experiences, practices, and identities. This article primarily makes use of the traditional methodological tools of production studies but aims to strengthen this analysis through engagement with the developing practice of hands-on historical research.

As the field of production studies has grown in recent years, so too has the number of methodological devices utilised by those engaged in this research. Principal amongst these tools are ethnographic fieldwork and oral history interviews which have become a staple for researchers of production practices and communities. These tools are valued for their ability to uncover the rich stories, or 'thick description', that detail the practices and technologies of production personnel, and provide a "sense of the ambience and texture of the industry's life as it is lived" ${ }^{\text {. However, }}$ 
such methodological tools are not without disadvantage. Observational fieldwork has been utilised by numerous high profile studies ${ }^{7}$ but, of course, is not currently an option for those engaged in historical studies. Oral history interviews often form the bulk of historical studies of production personnel. However, such accounts require careful scrutiny and a measured approach in order to yield successful analysis. John Caldwell ${ }^{8}$ has shown that research of this kind, particularly when heavily reliant on interviews, is hampered by the industry's obsession with overt self-reflection. In an industry that is awash with 'behind the scenes' content, industrial self-analysis, and promotional 'spin' it is increasingly difficult for researchers to cut through this cultivated image and observe professional practice as it is actually performed . Furthermore, Helen Wheatley ${ }^{9}$ has pointed to a number of methodological hurdles in television studies, one of which is overcoming nostalgia in recollection. Narratives distorted by mournful loss or nostalgic sentimentalism are rife amongst such recollections. Borrowing from Carr" ${ }^{10}$, Wheatley warns against the "romanticism of old men and societies" that abound within the retelling of past activities and cultures.

Production studies researchers therefore commonly support these methodological tools with further textual analysis of industry trade journals and archives. Trade journals are undoubtedly useful resources for many functions that are difficult to capture with oral histories, such as analysing the industry wide trends, precisely accurate timeframes, and capturing intricate technological detail. However, their use does come with certain limitations. Such publications are, by nature, outward facing and represent the public voice of a given industry or organisation. As such, they are undoubtedly susceptible to Caldwell's notion of truth obscuring industrial 'spin'. Furthermore, to maintain readership, such publications are often focused on new technologies, experimental practices, and fresh ideas. Unearthing the mundane daily practices of a given community from these publications is a difficult task.

Archives pose similar difficulties for those who wish to analyse the hidden practices of individual production communities. As useful and comprehensive as they may be, these institutional organisations seemingly struggle to capture the stories of individuals and present a significant challenge to those who seek information about distinct production communities or the intricacies of individual practices. As a result, they are of limited use when examining the unspoken and unspectacular skills, identities, and cultures of particular personnel. As Vanessa Jackson ${ }^{11}$ states,

These traditional institutions, for instance the seemingly comprehensive BBC Written Archives at Caversham, preserve the production files and documentation about how the broadcast centres were equipped, but there is little trace of the production practices, about how particular equipment was operated, or what a new piece of technology allowed you to do in terms of programme making, that its predecessor did not. It is the human interaction that is missing in the archives, which preserve only the tangible, physical artefacts.

Clearly, when used in combination, these imperfect methodological tools undoubtedly provide reliable, thorough, and compelling accounts of the work of the communities that inhabit production environments. However, there clearly remains some scope for developing methods that further support this existing methodological tool kit and sharpen the focus on certain aspects of this field. This article hopes to show how such methodologies can bring new research opportunities and add to the growing number of scholars advocating for more hands-on research within production,

7 John T. Caldwell, 'Breaking Ranks: Backdoor Workforces, Messy Workflows and Craft Disaggregation,' Popular Communication, 8, 3, 2010,

221-226. Vicki Mayer, Below the Line: Producers and Production Studies in the New Television Economy, Duke University Press, 2011.

8 John T. Caldwell, Production Culture: Industrial Reflexivity and Critical Practice in Film and Television, Duke University Press, 2008.

9 Helen Wheatley, ed, Re-viewing Television History: Critical Issues in Television Historiography, I.B.Tauris, 2007.

10 Edward Hallett Carr, What is History, Macmillan, 1990.

11 Vanessa Jackson, (2013) 'Revealing Television's Analogue Heroes,' VIEW Journal of European Television History and Culture, 2, 4, 2013 , 3-14. 
television, and media studies ${ }^{12}$ (Fickers \& Van den Never 2013; Jackson, 2013; Murphy, Aust, Jackson, \& Ellis, 2015, Hall \& Ellis 2019).

This article combines the traditional methodological tools of production studies with supporting hands-on research data generated by the work of the ADAPT project ${ }^{13}$. Throughout its 5 years, ADAPT produced numerous projects, known as simulations, which brought together retired television production personnel with the now redundant tools utilised throughout their careers. By doing so, these simulations provide an opportunity for hands-on research data to be generated by doing rather than telling and exposed by the professionals rather than by researchers. Admittedly, such an approach clearly has its own limitations. Despite the considerable efforts of the ADAPT team, no research project can truly recreate the historical environment in which these practices were originally performed. Furthermore, there is undoubtedly an element of performance and awareness of self representation inserted by each contributor. While significant work went into minimising this (such as the use entirely original technologies and recording the interactions using hidden fixed rig cameras) it is undoubtedly an inescapable aspect of historical research and something acknowledged by the ADAPT team themselves

These are not 'reconstructions' in the sense that they do not seek to reconstruct any specific past event. Nor do they seek to take the participants back in time to behave as they once did. ADAPT's simulations are acts of memory for the participants, but they are memory events which are quite unlike those which take place in an interview. The participants are asked to demonstrate what they used to do regularly: how their machines worked, how they worked together, what their routines once were. They are active collaborators in the research, living again their physical and emotional encounters with machinery that they used to use on a daily basis. They exchange information with each other as well as the filmers observing them, often struggling to recall specific and intricate details of their past working habits. ${ }^{14}$

These simulations also provided an opportunity to conduct ethnographic work through direct involvement in their production but also produced many terabytes of video data ${ }^{15}$, some of which has been analysed to draw out the hidden and unspoken practices of these communities. This hands on data has been utilised as a further approach to this subject, providing further clarity and focus to elements not well served by other methods. Simulation data is used in combination with more than fifty oral history interviews which form the basis of this research. This approach provides new insight into the hidden work and experiences of a community that has so far, been chronically under researched.

'Television sound operators' is of course a broad term that encompasses multiple and unique social groupings which do not necessarily share working practices or professional identities. Whilst it is a label that almost all interviewees for this research would identify with, and a useful term when addressing industry wide issues and trends, it should not be mistaken for a specific job role. As this article focuses primarily on those working on Outside Broadcast productions (OBs) , the main job roles of concerned are Sound Supervisor, First Sound Assistant, and Second Sound Assistant. However, where there is significant shared experience or overlaps in practice, reference may be made to those who worked $16 \mathrm{~mm}$ film sound acquisition, such as Sound Recordists.

12 Andreas Fickers and Annie van den Oever, A. (2013) 'Experimental Media Archaeology: A Plea for New Directions,' in Annie van den Oever, ed, Technē/Technology Researching Cinema and Media Technologies - Their Development, Use, and Impact. Amsterdam University Press, pp.

272-278; Amanda Murphy, Rowan Aust, Vanessa Jackson and John Ellis, '16mm Film Editing: Using Filmed Simulation as a Hands-On Approach to TV History,' VIEW Journal of European Television History and Culture, 4, 7, 2015, 7-10; Nick Hall and John Ellis, eds, Hands On Media History, Routledge, 2019.

13 ADAPT TV History, 'How television used to be made,' ADAPT: Researching the history of television production technology, June 2019, http:// www.adapttvhistory.org.uk/

14 Amanda Murphy, Rowan Aust, Vanessa Jackson and John Ellis, '16mm Film Editing: Using Filmed Simulation as a Hands-On Approach to TV History,' VIEW Journal of European Television History and Culture, 4, 7, 2015, 7-10.

15 ADAPT TV History, 'How television used to be made.' 


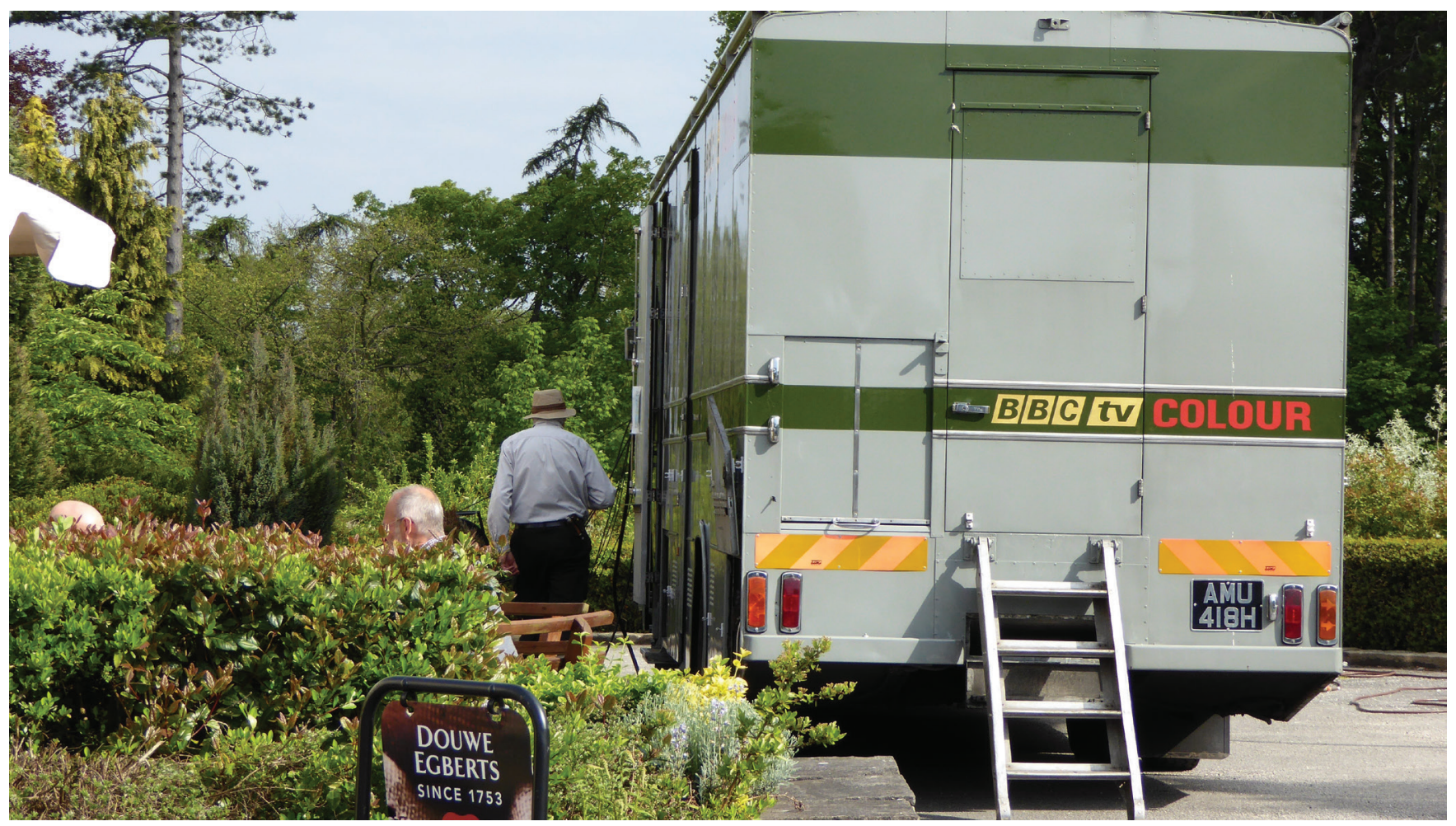

Figure 2. ADAPT's OB Simulation filmed in northern Wales.

\section{Outside Broadcast Sound in the Era of Vertical Integration}

In the 1970s, completing a successful outside broadcast was a significant technical operation. The OB truck, known as a scanner, contained everything needed for a self-sufficient mobile television studio and was the base for a crew of up to 15 skilled operators and production staff. Within the cramped scanner, the space was divided into three sections of which sound, alongside vision and production, occupied their own distinct area. This small space contained all the equipment needed to create programmes sound track including the mixing desk, a $1 / 4$ inch tape machine, and multiple patch bays. Due to the size and requirements of OB production, sound required a crew of 3 operators headed by a senior operator known as the sound supervisor. The second in command, who sat alongside the supervisor in the truck, was known as the first sound assistant, while support outside of the truck was provided by the junior member of the sound crew, the second assistant. Within this small team, there was a clear and defined hierarchy and, to enable this crew to complete all the work necessary in the short period of allocated time, all working processes were well-established and highly refined. From the initial set up, to mixing the sound during live transmission, everything was meticulously planned and performed with military-like efficiency.

For a run-of-the-mill $\mathrm{OB}$, the sound crew usually arrived on location on the second rigging day. Following the initial installation day, in which the 'riggers', also known as cable bashers, would position the bulk of the heavy multicore cabling, the sound team would arrive and begin their own sound-specific installation. The sound supervisor was responsible for the overall planning, installation, and delivery of the programme soundtrack. It 
was their responsibility to attend initial planning meetings with other senior personnel, such as the director and senior camera operator, and to liaise between the sound crew and other departments before and during broadcast. Unlike the other members of the sound crew, sound supervisors were not assigned to a particular OB truck, but were specialists in their chosen field of production, such as golf or football. Using this expert knowledge of their specialism, the sound supervisor would draw up a plan for microphone installation and delegate its implementation to the assistants.

More so than any other stage of the production, this installation process was complex, labour intensive, and relied upon a highly entrenched set of working practices. In the pressurised and time sensitive environment of live television, and with a relatively small team in charge of all aspects of the programme sound, it was crucial that sound operators were able to work quickly and harmoniously to install, test, and perfect the microphone setup. To enable this, the crew relied on working to established and universal procedures that had been developed through months of structured training and further engrained through years of on the job peer to peer learning and the communal sharing of processes and tacit knowledge.

These highly refined uniform working practices, or 'BBC way of doing things', was a common trend amongst those interviewees who had worked in OB production. Of course, during the 1970s, the BBC still functioned as a large vertically integrated organisation and, due to its considerable resources seemingly untouchable place within the comfortable duopoly ${ }^{16}$, was the dominant force in almost all aspects of British broadcasting. As the primary employer, and educator, of broadcasting personnel their organisational practices permeated the entirety of the careers of our contributors. Some interviewees discussed this in regards to their training, stating that, much of their initial training, centred on engraining the practices and cultures of the BBC into new recruited sound operators.

You'd go off to Wood Norton and learn the way the BBC done things. What they were doing was making sure if you snapped someone in two they'd have BBC written right through them like a stick of rock [...] Because we were all staff, from the producer, the director to the designer and the costume, we were all in the same building, so it was important to have that understanding (OB Sound Supervisor 9).

Others discussed these entrenched practices in relation to their activities when out on a production. One location sound recordist likened working on a $16 \mathrm{~mm}$ film shoot to that of a well drilled military unit.

[Going on location] Was like going out as a mini platoon, with the cameraman as the leader...The cameraman was the operator in charge, he was on a higher grade than the sound man, but we were definitely a team. (Sound Recordist 4).

Yet another interviewee touched on this, commenting on the "common purpose" and "common way of doing things" instilled into operators working at one particular broadcaster:

Everybody had a common purpose, everybody went through the same training stuff at Evesham [...] There were clashes obviously, there always is in those environments, but everyone was using the same sort of rules (Studio and OB Sound Supervisor 2). 
T. Heath, Television Sound Operators

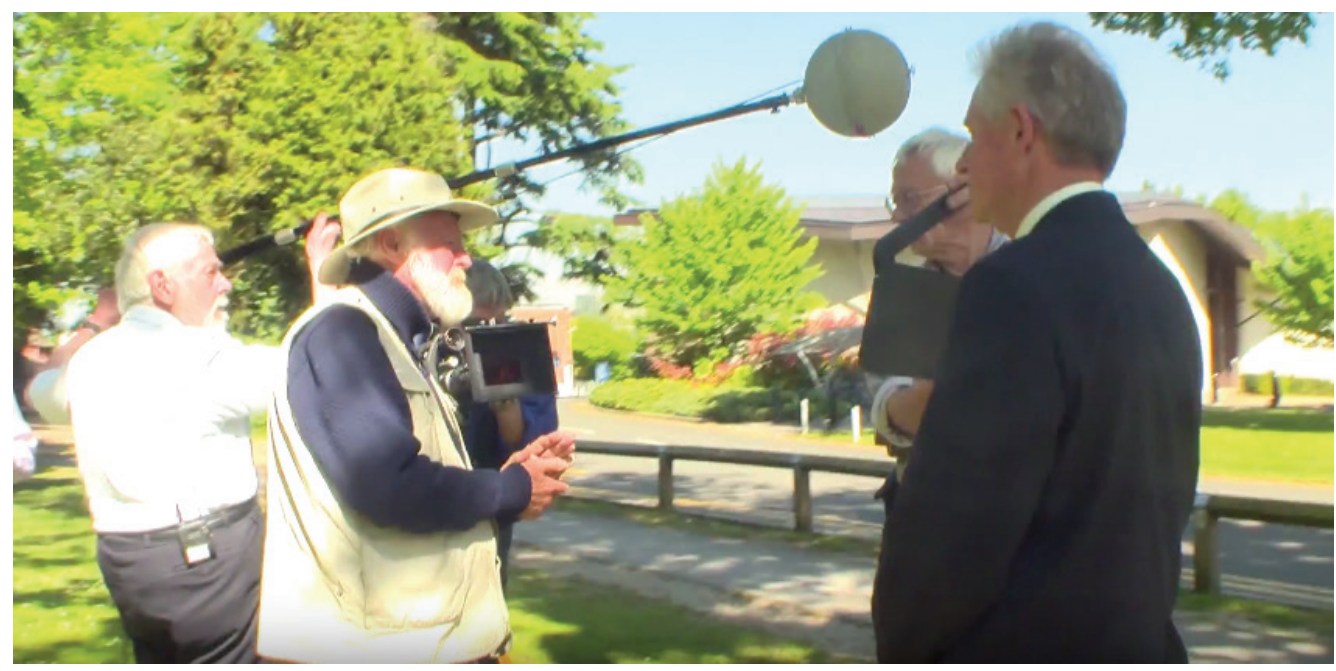

Figure 3. Reunited $16 \mathrm{~mm}$ film crew take part in simulation filming in the grounds of Royal Holloway.

Whilst these spoken accounts are undoubtedly persuasive, the most telling evidence of these entrenched practices can be found within the data from ADAPT's $16 \mathrm{~mm}$ film and outside broadcast simulations. During the $16 \mathrm{~mm}$ exercise, participants were tasked with setting up and filming a $16 \mathrm{~mm}$ documentary sequence centred around a seated interview. Within just twenty four minutes of accessing the set, the participating crew had re-familiarised themselves with and set up the 1960s equipment. Much of this equipment they had not touched for some decades, but in less than half an hour they were ready and waiting to shoot. During this set up period, numerous technically and artistically complex processes were worked through with great efficiency. Although working on their own distinct tasks and job roles, differing groups of operators as well as the director worked in sync with one another, aware of their the requirements and limitations of their colleagues' tools and practices. Utilising the practices and skills engrained through decades of experience, with only minimal discussion regarding the technicalities of the setup, the contributors were able to set up the camera, lighting, and sound for the shoot in this short space of time. This shoot, a sit down interview in doors, was undoubtedly a fairly modest setup for a veteran television crew. However, the same process can be observed in the events of a much more technically ambitious production. Throughout the ADAPT's simulation of a 1970s sports OB the hierarchies and highly established working relationships within the sound crew were again clearly illustrated by the contributing operators.

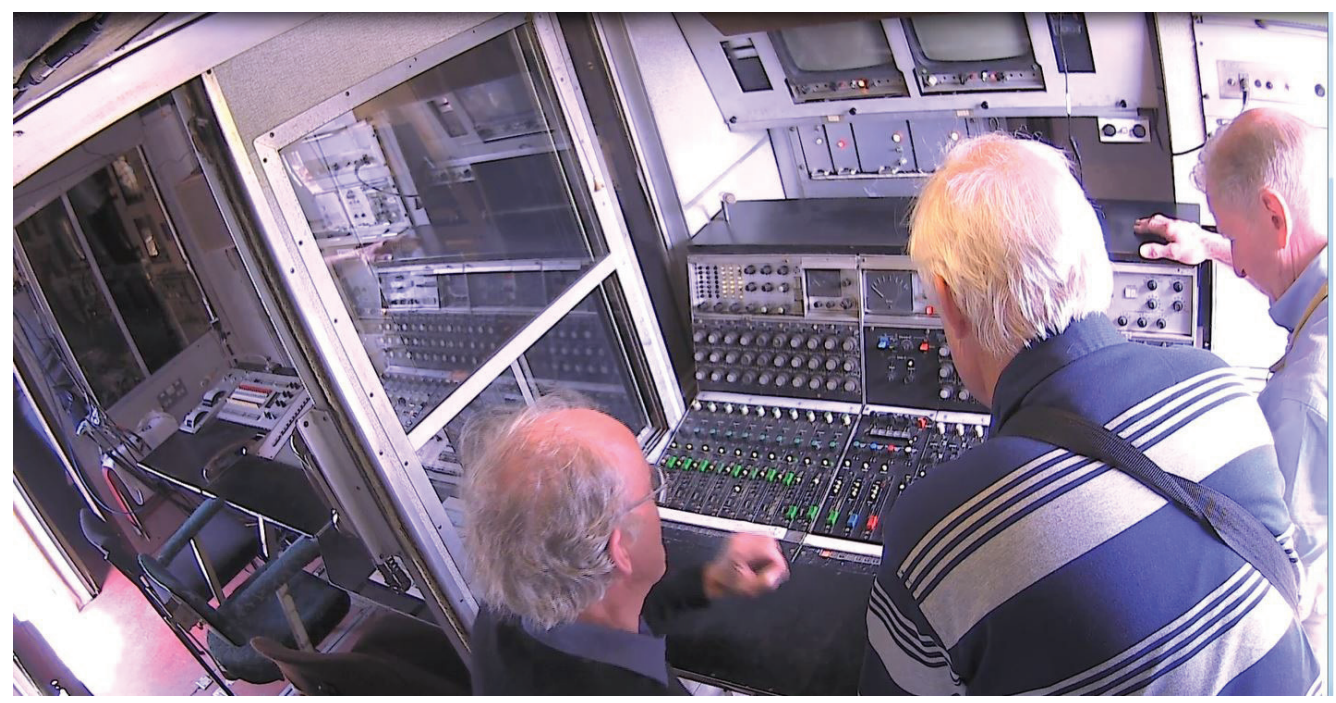

Video 1. The team of $\mathrm{OB}$ sound operators are reunited with the mixing desk used throughout their careers. Here they discuss their approach to the production of ADAPT's OB simulation. 
As shown in this clip, the contributing sound supervisor, sound assistant, and second assistant seamlessly reverted back to the workflows and hierarchies of their previous careers, despite the many years that had passed since their respective retirements. At the start of the installation day, the sound supervisor met with the sound assistant and second assistant in the sound area of the scanner to brief them:

Sound supervisor: Alright if you could both come round to the back of the scanner [...] Okay, so basically it's going be a commentator on a lip mic, an effects mic, which I think we have an 816 for general effects. And we need a little mic behind the dartboard to pick up the darts going in. And a stick mic so if there is any in-vision stuff or interviews, we can deal with that. So that's four pairs on the multi-core, the 7 way cable is going in now.

Sound assistant: I think we have two multicores we can use.

Sound supervisor: "Two multicores! What a luxury, ok then, one for programme and one for comms. Now, at the moment we don't have any cables, we're waiting for them to be rigged, so I'm going to refamiliarize myself with the desk. If you could take a few of the mics out [sound supervisor hands mics to the assistants] that can go outside, keep it all together, mind your step and l'll test the desk.

This briefing demonstrates not only the clear hierarchy amongst the sound crew but also the highly established nature of their practices. While the sound supervisor does outline an overall plan, they omit any detailed instructions as they are simply unnecessary. Some of these generalised instructions, such as "one [multicore] for programme and one for comms", require significant knowledge and training just to initially decode. Acting upon these instructions involved significant planning, rigging, and testing. However, short instructions could be given for these highly complex tasks due to the fact that the processes involved were well established and the practices of the crew were well refined. The manner in which this exchange takes place is also significant. Unlike the interviewee accounts, which contain much emotive and dramatic uses of language, this discussion, was brief, casual, and done away from the main action of the day, only captured by the fixed rig cameras seemingly unnoticed by the contributors. Clearly, for those taking part in this simulation, this exchange did not warrant closer scrutiny or supplementary explanation. It was simply a trivial mundane working process to be completed before the installation work began. However, as shown, this coded discussion was vital to the installation process, and by extension the entire sound operation, and was crammed with unspoken expert knowledge, skills, and expertise. This small section of hands-on data, provides an insight and a context to the interview data that is simply not appreciable in the oral history data alone. Use of language, and the inherent difficulties of oral histories, can often apply new meanings, and unwarranted significance, to historical processes. This hands-on approach gives a new visibility to these processes, drawing them out through more organic interactions between contributors. Much like the "thick description" advocated by Geertz ${ }^{17}$ in oral history research, the hands on nature of these interactions go beyond listing the technologies and practices but displays the wider context in which they occur. The use of simulation allows research to be conducted through observation and viewing allowing the researcher taking a less obviously leading or supervisory role in the extraction of the data they wish to analyse. Rather than pushing contributors to consciously provide thick description, hands on history displays the "ambience and texture of industry's life" through hidden practices in their most mundane, but realistic form. 


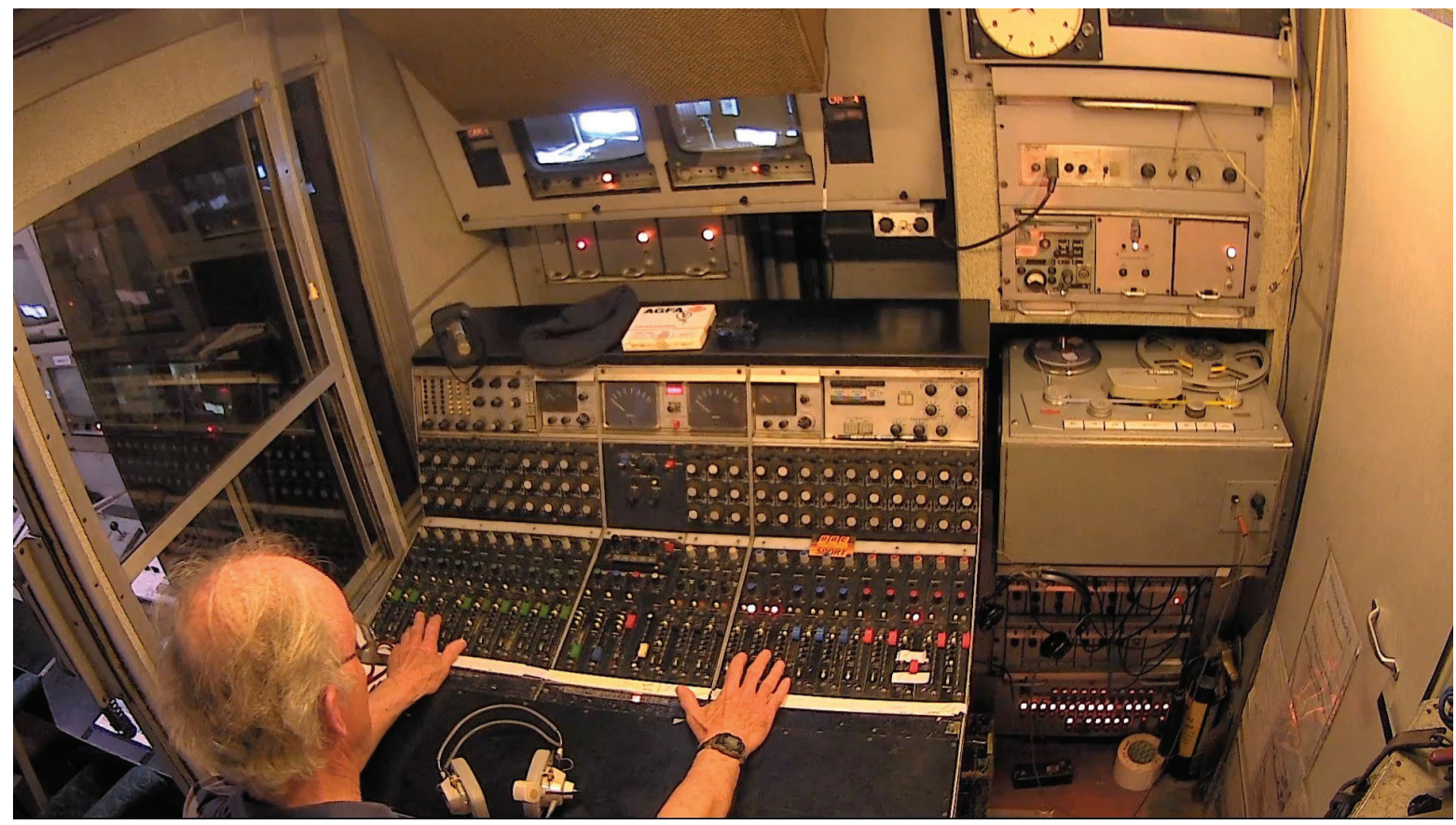

Figure 4. Analogue OB Sound desk within the original Type 2 scanner.

Following the initial planning and briefing, the sound supervisor would set up the numerous input and outputs of the mixing desk. While this was not a uniform arrangement, general rule of thumb dictated that the first ten channels were taken up by commentary and other dialogue and known as green tracks, followed by five channels of effects mics known as red tracks and a number of spare channels occupied by additional mics known as blue tracks. Finally a channel was dedicated to the tape machine, from which music and effects could be played in the mix. The actual layout of the tracks was set by the sound supervisor and depended on how the incoming channels were patched into the desk inputs.

Until the early 1970s, the BBC was at the forefront of the development of production technologies and for a significant period designed and built its own desks, foremost of which was the Type D. However, by the mid 1970 s the BBC had begun to dispose of their in-house manufactured mixing desks, including the Type D and sought replacements from external companies. While, initially, Pye Ltd desks were used for OBs, by the end of the 1970s, Neve had become the manufacturer of choice, not only at the BBC but also in the wider industry. Regardless of the manufacturer, these desks were all analogue mono desks with, usually, 24 available channels. While an entire programme would usually be recorded on 24 channels, it was not unheard of for a small external six-track mixer, with a single channel mono output, to be used to expand the capacity of the main mixing desk. The following decades saw significant developments in mixing desk technologies, most notably the integration of automation and digitally assignable desks. However, due to the live nature of OBs, the advantages of these desks were mainly found in the flexibility of their installation and setup, rather than their actual operation during transmission or recording. When mixing sound live, or as live, the sound supervisor would have had to manually operate all tracks simultaneously, following the unfolding action and taking instruction from the director, regardless of the affordances of the desk. This ability to follow and anticipate the event being covered was crucial, as a sudden spike in sound levels, such as that caused by crowd reaction, not only sounded unpleasant but also had the potential to cause a host of technical issues, such as distortion. Consistent failure to predict peaks, and adjust the levels appropriately, could lead to a programme being taken off air if it breached strict broadcast volume level rules, while, in extreme cases, exceptionally high peaks could blow the transistors in the OB truck. Therefore, the ability to effectively manage a mix and react appropriately to the unfolding live events was at the heart of the sound supervisor's work. 


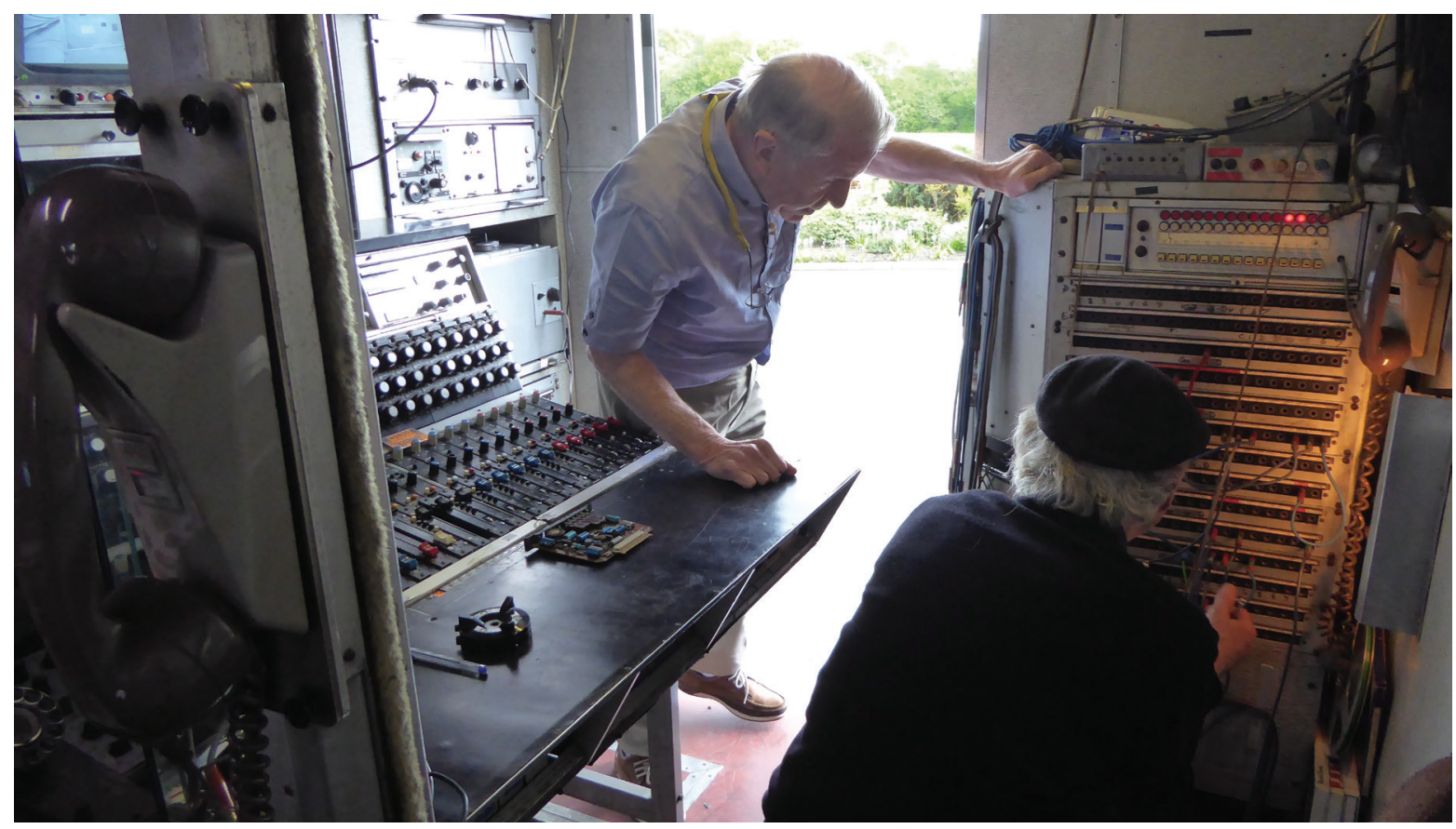

Figure 5. Ken Osbourn and Doug Whittaker work over the patch bay used to connect the numerous inputs and output within the OB scanner.

Whilst the title sound assistant may seem a modest role, the role of this operator was crucial to a successful broadcast. The role of the sound assistant had two distinct aspects. During shooting they would assist the sound supervisor with the task of mixing, often controlling a number of faders if the programme required a high number of tracks. The sound assistant also had a number of tasks during transmission, including cueing and playing-in sounds or music from $1 / 4$ inch tape and operating jack fields. However, the most significant element of the sound assistant's work came before any rehearsals or live shooting had even taken place. During the setup phase, the sound assistant was responsible for planning and implementing all local communications and talkback for the entire outside broadcast. These processes were of the utmost importance, as they enabled separate departments to communicate with one another and, crucially, allowed the director to orchestrate the numerous distinct elements that make up an OB into a cohesive operation. Working off the large multicore cables installed by the riggers, the sound assistant would provide each required destination with the necessary "comms lines". Destinations, such as the commentary box, camera positions, and studio area, would need to be provided with a number of separate lines in order to operate efficiently. An initial feed to the sound output, known as a music line, was provided, along with a talkback line, which allowed the scanner to communicate with the destination, while a further reverse talkback line, allowed the destination to communicate back to the scanner. Implementing this complex communication system, often in less than ideal conditions, took considerable planning and skill to successfully complete. As one sound supervisor, who had worked his way through the second assistant and assistant ranks, describes:

Rigging the comms was often far more complex than the actual sound mix, people would often ask why we had so many outputs, well the reason was for all the comms" (Sound Supervisor 3).

Another stated:

I cannot emphasise enough that in sound operations, communications ranked equal with audio in making productions run smoothly. [Because of] The operational nature of the overall job and since the inputs and outputs to the communications systems were often in the domain of the sound operators, the logical conclusion was drawn that sound should devise and deliver both communications and audio. (Sound Supervisor 4). 
As a sound assistant simply adds:

A huge part of our job was to make sure everyone heard everyone else!

(OB Sound Assistant 2).

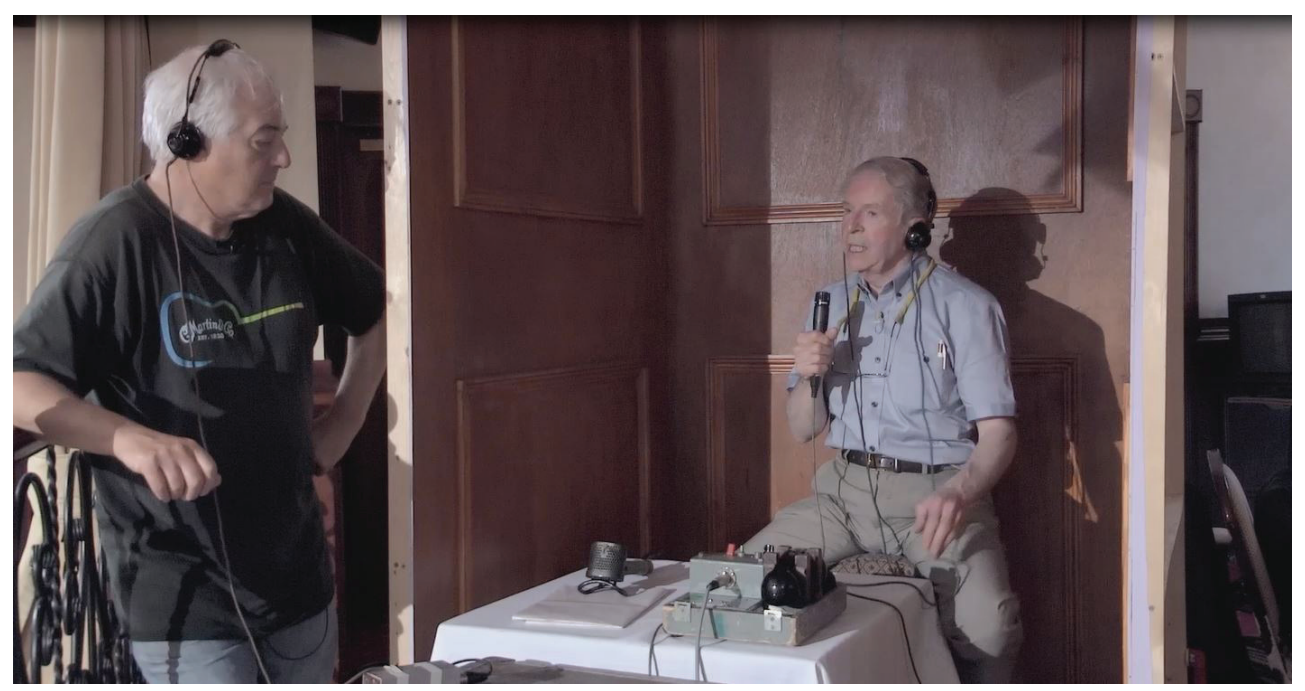

Video 2. Ken Osbourn and Doug Whittaker discuss the role of communications in an OB shoot.

In this clip, Ken Osbourn a retired BBC sound supervisor, discusses and demonstrates the importance of good communications within an $\mathrm{OB}$.

As with the interviewees quoted above, Ken stresses the importance of communications in allowing the director to communicate with the other departments from his position within the truck. Whilst the significance of these practices are undoubtedly clear from the recollections of Ken and the aforementioned interviewees, the true extent of the importance of communication systems in $\mathrm{OB}$ production is more fully revealed when seen in action. The following clip from Adapt's OB simulation demonstrates the 'live' transmission process of a small darts tournament, something that would have been considered 'bread and butter' work for a BBC OB crew.

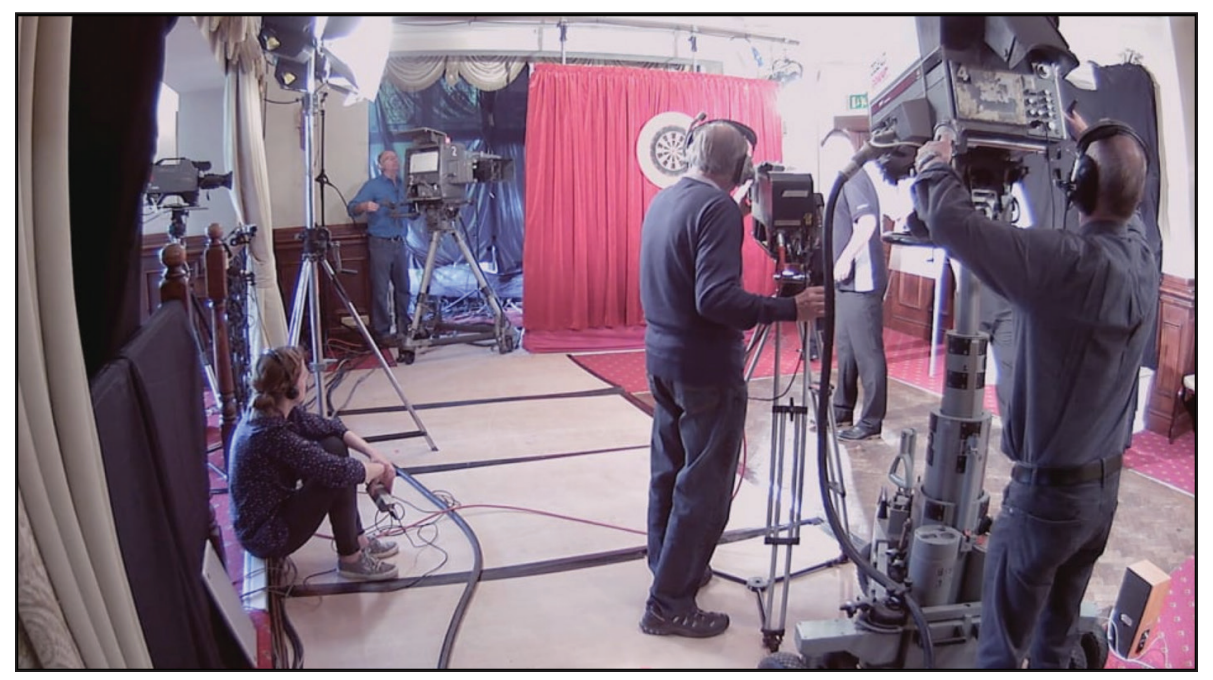

Video 3. 1970s OB transmission process. 
Throughout this clip, the director, Geoff Wilson, gives commands and direction to the numerous separate operators and departments. From the first second of the entrance music, until the final roll of the credits, Geoff is constantly orchestrating the production from his seat in the truck and coordinating many extremely complex processes. From instructing the VT operator to queue a replay, to directing every camera movement, and even shepherding on screen talent around via the floor manager, the necessity for Geoff to be able to quickly and effectively communicate is clear to see. Without appropriate communications in place, the entire transmission, although quite mundane by $\mathrm{OB}$ standards, would simply fall apart. As discussed, the significance of the sound assistant's contribution was stressed consistently within the interview data. As such, the hands-on data within these clips may not directly display any new or unknown information. However, it does clearly provide greater context to these practices than can be found in the interview data alone. It is a commonly cited hazard of oral history data that individuals are prone to aggrandise their own contribution, role, or status within such retellings. However, the footage from this simulation shows these processes in a form that reduces subjective interpretation and personal meanings. It displays them simply as routine processes conducted as they would have been day in and day out by a working OB crew. This clip shows how a hands-on approach to historical studies of production can allow researchers to bypass many of the shortcomings inherent in more traditional methodologies.

Assisting the sound supervisor and assistant from outside the truck was the job of the junior member of the crew, known as the second assistant. While the initial cabling was set up by riggers a day before the sound crew arrived on location, second assistants were responsible for setting up microphones and connecting them to the web of existing multicore cables. They would also play a crucial role in testing and fault-finding after the initial microphone installation, testing individual microphones while the sound supervisor checked for a signal at the relevant desk input in the scanner. Depending on the outcome of these tests, the second assistant, working alongside the riggers, would either find and replace the faulty cable or begin positioning microphones and securing cables to be used for the duration of the shoot. During the shoot, the second assistant would be armed with back-up microphones, ready to act should anything fail outside of the truck.

Unsurprisingly for this type of production, managing the vast and complex technologies, avoiding failures, and having adequate contingencies were the predominant concerns of those working on OB sound. In the era of analogue technologies, failures were commonplace, meaning that the sound crew simply could not rely on their equipment to work faultlessly throughout a shoot. As such, planning around these failures by ensuring sufficient backups were in place, as well as staying on top of general maintenance, was crucial to the successful operation of a programme's sound. Added to this technical frailty were the inherent pressure of live, or as-live, television and the numerous difficulties of working on location, often in less than ideal conditions. For outside broadcast crews, major sporting events, royal appearances, political summits and a host of other significant public events were considered everyday work. Due to the nature of such events, the ability to capture good quality sound at the first and only attempt was essential. Discussing their approach to work and priorities when on location, multiple interviewees pointed to the importance of managing their equipment and building-in sufficient space to absorb faults within their setup, as one sound supervisor stated:

In the days of type 2 scanners the focus was on the engineering and making things work. The technology at the time just could not be relied upon, so having adequate backs ups, and completing adequate tests, were always the key priority for the sound department. Other considerations included the weather: all kit was susceptible to damp, particularly connectors on cables. Half of the time was spent making things work and fault-finding (Sound Supervisor 5).

A sound assistant adds to this account, pointing to the importance of expecting and building equipment failures into initial work plans:

You always had a back-up mic to hand in case something went wrong, and if it did, you could switch to it, it might mean a few seconds of no sound but nothing that audiences would really complain about. You never left anything to chance. If you were doing something like a royal wedding you had 4 back-ups! [...] Generally, you'd build in a back up, so if you set up 32 lines, you'd be happy if 25 worked. A bit like cameras, they might have 5 cameras set up but they'd be happy if only 4 worked (Sound Assistant 3 ). 
T. Heath, Television Sound Operators

Another sound supervisor simply stated that his biggest concern was "well, that everything actually worked!"

However, whilst the overriding importance of technical proficiency was stressed by many, working in an OB sound crew was not a purely technical exercise. Hidden amongst these technical processes were moments of genuine creativity, experimentation, and artistic sensibility. While the fundamentals of microphone placement were generally formulaic, multiple interviewees discussed a number of incidents in which they broke this formula, going to great lengths in the pursuit of more complex and interesting sounds. As one sound supervisor stated:

The exec producer of cricket came to us and said "Can we put a radio mic in the stump?" This is before they had cameras in the stumps, before all the miniaturization and we decided no we couldn't get a mic small enough [to place in the stump]. So [...] at the BBC sports club ground at Motspur Park, we proved the case of burying radio mics. And it was used and, at his request, having seen the test that we showed him. [...] It did work but, as I remember, because it was rather damp at the beginning [it was not high quality], but, when it dried out it worked quite well [...] and then all the miniaturization came along and its been all improved, so it's no longer required (Sound Supervisor 6).

Another sound supervisor discussed how, as a newly recruited second assistant, he was tasked with suspending a microphone underground in order to achieve the desired bass-heavy tone of a firework display:

There was a lot of burying mics here and there. One of my first jobs was doing this international firework competition at Windsor Castle. And the sound supervisor said to me "I want a 1 metre square hole, cover the top with some bits of wood and put the turf back once you've suspended a mic in the middle". So I went off to the riggers and borrowed a spade and started digging. It actually sounded quite good, it gave this wonderful bass tone to the fireworks (OB Sound Supervisor 2).

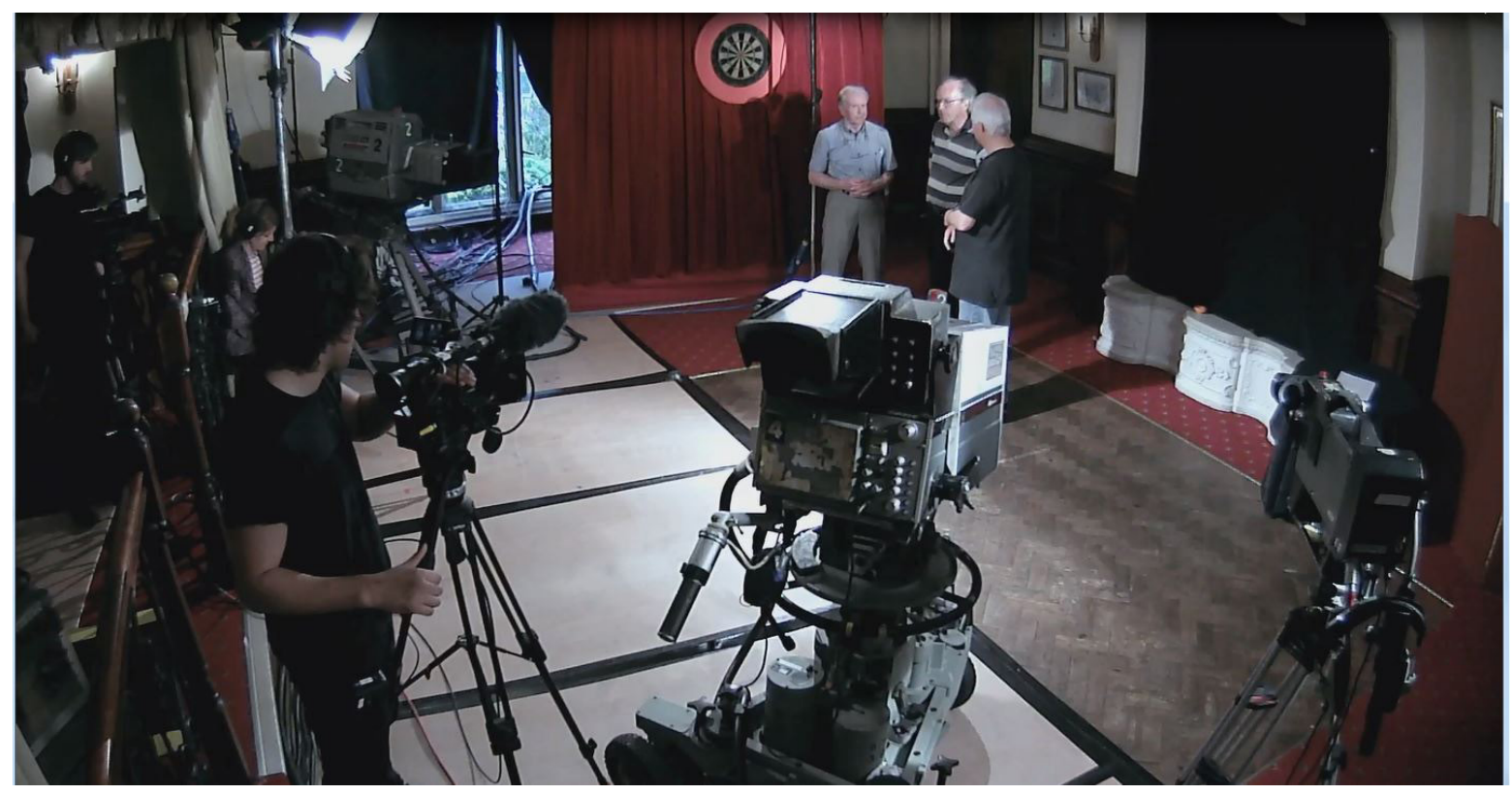

Video 4. Doug Whittaker and his team discuss the setup required to provide a "darts type atmosphere" for viewers at home.

During the rigging process for the Adapt OB simulation the sound crew offer a demonstration, and discussion of, their approach to microphone coverage of a simple darts tournament. The sound supervisor, Doug Whittaker, starts the demonstration by discussing the microphones used to gather sound effects for the programme. Here, Doug discusses 
T. Heath, Television Sound Operators

constructing a "darts type atmosphere" through the use of a long range effects mic to pick up the applause and noise of the crowd. During the transmission phase, Doug blends these sounds in with other effects, music, and of course commentary, to provide a more complex and immersive feel to what is otherwise a fairly unimaginative soundtrack.

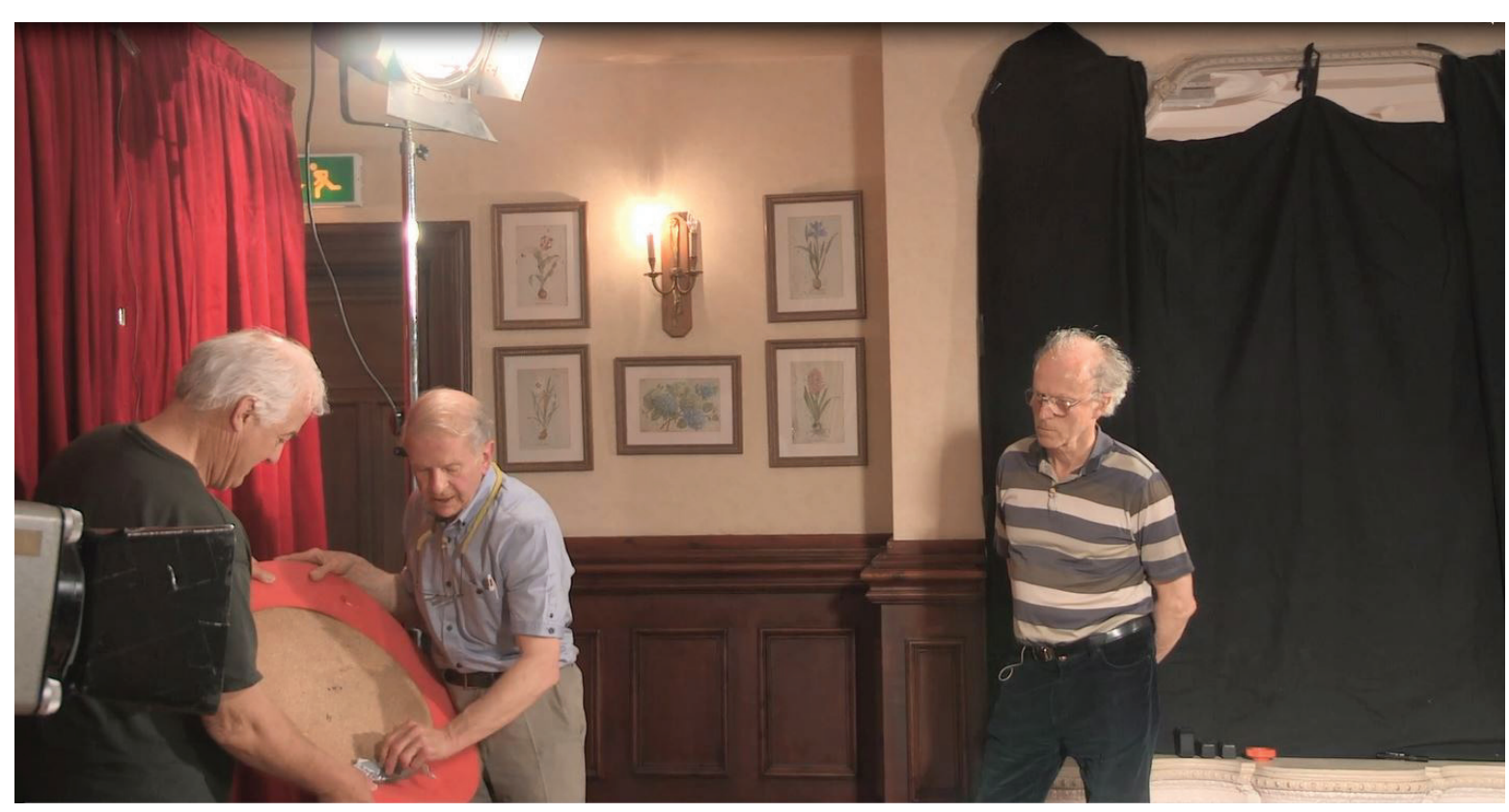

Video 5. Ken Osbourn demonstrates the use of miniature microphones to record effects as used in the 1970 s.

Also discussed in this clip is the use of a Sony ECM50 microphone hidden behind the dart board to capture, in Doug's words, the "really nice [...] thump of the dart as it hits the dart board". This is demonstrated later in the clip by Ken Osbourn, acting as a sound assistant, and displays the crude experimental nature of such creative practices. Far from being a sleek or complex process, a small personal mic, usually attached to clothing to capture speech, is wrapped in large chunk of blue-tack and taped directly to the back of the dart board. This process is undoubtedly a fairly simple one, and one that may not appear to be a particularly impressive example of creative expression. However, during an OB shoot, sound was one department in a much larger production crew working on a format in which the visuals take priority. As such, the sound crew had to conduct such artistic and creative practices around the practices and requirements of the other crew members. Despite the limitations of this creative practice, it undoubtedly held significance to the work and identity of those working in the pressurised, hierarchical, and technologically complex environment of $\mathrm{OB}$ sound.

Doug quickly moves on to discuss the more utilitarian aspects of sound such as the commentary and communications setup. Although a brief discussion, it clearly demonstrates that contributing to the artistic merit of the programme was a genuine concern for the sound crew. Programmes such as darts or cricket coverage would have been perfectly adequate for broadcast without the added sound effects that these processes provided. However, as evidenced by the interviewees' and Doug's team, experimentation and the application of creative processes was an important aspect of their professional practice. What is also clear from this clip is the use of hands-on research in unearthing the hidden day-to-day practices of production personnel which are easily lost in other forms of historical investigation. The practices discussed and demonstrated within the footage are not spectacular or unusual but are the daily processes conducted by sound crews when covering a programme considered to be 'bread and butter' work. This is in direct contrast to the data produced by the interviewees quoted earlier. These interviewees discussed creative practice in relation to events outside of their normal daily practices, such as the sound supervisor who was approached by a senior producer to devise new methods for covering cricket matches. Such recollections centre around extraordinary 
or unusual practices conducted during highlights, or at least noteworthy times in their careers. Whilst such recollections are undoubtedly important and worthy of analysis it is clear that they can act to obscure the true daily reality of those working in these roles. As discussed, throughout their daily working lives sound operators were not given great time and resources to experiment with sound acquisition at their leisure. These practices took the form of easily assembled non-disruptive experiments implemented by quick thinking sound supervisors and could only be implemented where the practices of their colleagues accommodated them. Once again, hands-on research shows its worth in uncovering this daily reality in its most authentic, and unspectacular form.

\section{Conclusion}

This research has made use of hands-on methodologies to support and improve analysis of a small yet important group of television production personnel. The combination of hands-on research with traditional oral history interviews provides greater insight into this group than can be gleaned from traditional methods alone. Oral history accounts have signposted the broad issues significant to those who make up this community. Hands-on research has allowed for analysis of these accounts to be tightened and to home in on the granular detail that is often hard to draw out in oral histories. Data from the ADAPT simulations has uncovered the unspoken practices of this group and reveal their unspectacular, day-to-day practices. While isolated oral history recollections often focus on the significant or unusual events, and are prone to embellishment from even the best of contributors, the simulation data lays out ordinary practices to see first hand. Furthermore, hands-on methods more easily allow for researchers to extract 'thick description' eagerly sought in oral histories research. By changing the research dynamic, allowing the technologies and situations to push the work forward, researchers are more easily able to sense the context and historical specificities at play. By uncovering the sense of the industry and working practices, research of this kind can more readily contribute to the wider field of television studies and inform our theoretical approach and understanding of television output.

This article has analysed only a few aspects of the work of television sound operators. Within outside broadcast production alone, there is multiple practices and skills not covered in this work. Furthermore, outside broadcasts is only one form of production, outside of which there are numerous skilled sound operators in different job roles and departments. Despite this limitation, significant new understanding about this group has been gleaned from this analysis. On an outside broadcast, the sound crew was a small but crucial team of highly skilled operators working with unreliable technologies often in hostile environments. ,The unique requirements and implications of outside broadcast production greatly impacted on, and shaped, their practices and professional identities. Not only did these professionals have to work efficiently together but also, as a team, they had to fit into the wider hierarchy of the entire crew. Due to these unique characteristics of OB production, the sound team was required to maintain its own strict hierarchy and to be able to work harmoniously on separate aspects of the sound and from multiple locations. To enable this, they relied on a set of routine, highly refined skills which were engrained into their daily working practices. They were able to work through extremely complex issues with minimal discussion utilising tacit knowledge and shared understandings. Furthermore, the technological frailty of their equipment as well as the pressurised environment of working in a large crew with limited time, impacted on their work and refocused their practices. For sound operators the primary focus of their work was on maintaining equipment and utilising technical knowledge to ensure good quality programme sound. However, their work was not a wholly technical role devoid of creative practice. Sound operators went to great lengths to experiment with sound, and create a more complex soundtrack, where possible. Such opportunities were limited and existed around the borders put up by the wider crew however; they did hold significance to those working as sound operators and contributed significantly to their professional identity. The use of hands-on research has revealed a number of hidden and unspoken processes which in turn may provide insight into our theoretical understanding of sound and its role in television. Had this research relied on just a single established methodological approach, this new insight would undoubtedly have remained unknown. Clearly, production studies have much to gain from embracing such methods. 
T. Heath, Television Sound Operators

\section{Acknow ledgements}

This article and the footage it refers to were created as part of the ADAPT project funded by the European Research Council (ERC) under the European Union's Horizon 2020 research and innovation programme (grant agreement No 323626).

\section{B i o graphy}

Tim Heath is an independent researcher whose work focuses on television sound and the wider television production industry in Britain. He earned his PhD, 'Making TV Sound', from Royal Holloway in 2017 working as part of an EU funded research project Adapt TV History. 